\title{
LOS CAMINOS Y CARRETERAS EN ALICANTE DURANTE LA RESTAURACIÓN (1875-1902): CONSOLIDACIÓN DE LA MALLA VIARIA Y TRANSFORMACIÓN DEL PAISAJE
}

\author{
Jesús Rafael de Vera Ferré
}

\section{RESUMEN}

La política viaria en la provincia de Alicante durante el período de la Restauración se caracteriza por una consolidación de la malla básica y secundaria, de forma radial y arborescente, siguiendo criterios continuistas respecto a las realizaciones llevadas a cabo en la época isabelina, reforzando el papel hegemónico que desempeña el ferrocarril.

Las mejoras que se introducen en los caminos y carreteras de la provincia de Alicante ayudan a introducir las estructuras agrarias en los circuitos capitalistas, lo que conlleva transformaciones importantes en los paisajes, a la vez que se obtienen mayores plusvalías en la comercialización de los productos, lo que a la larga, va a devenir en la aparición de un proceso de industrialización. La articulación del territorio pone las bases también para un reforzamiento de la capitalidad de la ciudad de Alicante que se convierte en el núcleo principal de intercambio de los distintos medios de transporte.

\footnotetext{
ABSTRACT

The Restoration period presents a favourable balance respect the building and consolidation of tracks and roads network of the province of Alicante.

Once obtained making firm the big communications axis, whose principal location remains centralized across the city capital, the arduous task of making the network dense is working, what has repercussions over demographic and economic studwork of the area, being translated in profound changes in geographic landscapes.
} 
The profound transformations that the road and train introduce in the agrarian production, with cultivations changes, and in commercial bussiness, making it dynamic, will lead, lengthwise, in an increasing monetary flow towards industry.

The modernization of terrestrial communications system contributes this way, in remarkable form, to put the basis for the installation of provincial territory in capitalist circuits.

\section{Hacia la consolidación de una red viaria centralizada}

La política viaria durante la Restauración en Alicante presenta continuidad respecto al período isabelino y coadyuva a la paulatina introducción de las estructuras agrarias en los circuitos capitalistas y a una obtención de mejores plusvalías en la comercialización de los productos, lo que, a la larga, va a devenir en la aparición de un proceso de industrialización en algunas comarcas (1).

Los profundos cambios estructurales de la economía van a suponer un aumento de las bolsas de paro, paliadas en parte con la realización de obras viarias que crean y consolidan una malla radial y arborescente (2), solucionando de esta forma la deficiente situación existente descrita por Echevarría (3) y Orozco (4), el cual nos indica que, en los años iniciales de la Restauración, Alicante ocupaba el onceavo lugar entre las provincias españolas en cuanto a cantidad de carreteras y caminos, con una densidad de $83 \mathrm{~km}$. por cada mil kilómetros cuadrados de superficie territorial (5), que salvo en el caso de los grandes ejes provinciales e intercomarcales, que tenían un carácter carretero, estaban constituidos, en su mayoría, por caminos de herradura, en unas condiciones de conservación penosas, que se agudizaban en épocas de lluvias.

El esfuerzo constructor fue notable. En 1881 las vías estatales sumaban $352,867 \mathrm{~km}$. y las provinciales 72 (6); en 1888, ascendían a 495, 154 y 91,187 (7); en 1898, 624,685 y 145,618 (8); y en 1901, 638,558 y 159,355 km. respectivamente (9).

De esta forma, al iniciarse el Novecientos, la situación viaria de la provincia era ya bastante equilibrada. Los cuatro grandes ejes que convergen en la capital desde Alcoy, La Marina, la meseta, y Orihuela están plenamente en funcionamiento, mientras que la vía litoral hacia Torrevieja solamente está en construcción en el tramo Alicante-Altet.

En La Marina, la carretera que une Gata con Jávea está en funcionamiento (10), mientras que la que discurre entre Pego y Benidorm, por Callosa d'En Sarriá, solamente está abierta en los tramos Pego-Sagra y Bolulla-Benidorm, estando en construcción el de Sagra a Parcent y en estudio el de Parcent a Bolulla.

Los enlaces de La Marina con la Montaña cuentan ya con la importante vía que une a Denia con Cocentaina por Pego, mientras que está en construcción el tramo de Alcoy a Benilloba en la carretera que uniría la capital del Serpis con 
Callosa y el de Planes-Almudaina en la que enlazará el primer núcleo con Relleu.

El enlace de la Hoya de Alcoy con Villena, a través de Ibi, esta totalmente en servicio.

En el Campo de Alicante, estaba aprobado el proyecto de carretera directa entre la capital y Campello; concluido un tramo de la de Busot y otro en construcción; en pleno funcionamiento la de San Vicente, estando en obras el tramo hacia Agost, mientras que el trazado hacia la Hoya de Castalla, en dirección a Tibi, solamente contaba con el proyecto aprobado, situación que compartía con la proyectada entre San Vicente y San Juan, por Villafranqueza y Tángel.

El valle del Vinalopó tenía concluida la vía de Aspe a Santa Pola por Elche, la de Monóvar al límite provincial por Pinoso; la de Casas del Campillo a Villena y el enlace desde Novelda a la Vega Baja, por Aspe, Crevillente y Dolores.

En el Bajo Segura estaba finalizada la de Torrevieja a Balsicas y la que unía dicha vía con Orihuela, a través de San Miguel de Salinas y Bigastro, contaba con proyecto aprobado que discurría entre este núcleo y Benejúzar.

\section{La transformación de los paisajes agrarios}

La consolidación de la malla viaria, a pesar de sus carencias y deficiencias, permite el funcionamiento de una economía instalada en los circuitos capitalistas, aunque el protagonismo principal en los transportes corresponda a los trazados ferroviarios que aseguran la interconexión con los mercados exógenos, permitiendo la llegada de materias primas para el abastecimiento industrial y de los productos agrarios a los puertos para su exportación.

La carretera va a ir incorporando paulatinamente nuevos territorios agrícolas a una economía comercial exportadora, con una imparable tendencia al monocultivo de alta rentabilidad y especialización. Este hecho era vital para la burguesía e impregnaba todos los aparatos de poder desde la época isabelina.

Así, por ejemplo, en el Marquesado se produce un perfecto sincronismo entre las mejoras notables introducidas en su entramado viario y la profunda transformación del paisaje agrario, basadas en la expansión de la uva monstrell, lo que supone la cristalización de una auténtica fiebre pasera según Vargas (11) y la introducción en el valle de Pego de los cítricos, aprovechando la existencia de un suelo agrícola de buena potencia, acuíferos y buenas comunicaciones con los puertos de Denia, Oliva y Gandía.

Esta situación provocará una acumulación notable de capital entre los comerciantes británicos de la zona.

En la Vega Baja del Segura también se asiste a un doble proceso de mejora agrícola y del transporte, no solamente haciendo aumentar los beneficios agrarios en la zona de huerta, sino también afianzándose los gérmenes de una 
industria conservera de frutas y pimientos molidos, destinada en parte a la exportación.

\section{Alicante ciudad, centro de la malla del transporte}

Los beneficios provenientes de la agricultura comercial de exportación, especialmente en los sectores del vino, las pasas y los cítricos, se dirigen parcialmente a la creación de nuevas industrias aprovechando la coyuntura favorable de la mejora generalizada de la red viaria provincial, posibilitando la creación y consolidación de un auténtico mercado interior, especialmente con motivo de la firma de los Tratados Comerciales con Francia, en 1882, que supone la apertura de un período de expansión en la construcción de carreteras y caminos, un avance decidido del viñedo y una inusitada actividad del puerto alicantino que en 1880 había sido declarado de interés general de primer orden.

La ciudad de Alicante, convertida en el centro máximo organizador del transporte en el Sur del País Valenciano, debido a la acumulación de infraestructuras de transporte -puerto, ferrocarril y carreteras principales-, inicia una cierta industrialización de la mano de la clase burguesa, alguno de cuyos nombres más destacados son los hermanos Fourcade, Tomás Aznar Domenech, Valeriano Bosch y los señores Bono, Torras y Ferrer. A la fábrica de tabacos se han ido uniendo otras actividades, tales como dos refinerías de petróleo, fundición de hierro, aserraderos de madera, fabricación de hielos, construcción de cerámicas y ladrillos, elaboración de sacos, la tonelería o las industrias alimenticias de conservas, chocolates y pastas para sopa.

En la comarca que centra la capital, Agost tiene importante producción cerámica, mientras que San Vicente produce yesos y Jijona ve mejorar sus circuitos comerciales de distribución del turrón y de la industria del hielo con la carretera de Alicante a Játiva.

Al calor del crecimiento económico se desarrollaba la demografía de la ciudad que de 34.926 habitantes en 1877 había pasado a 50.142 a principios del Novecientos (12).

La movilidad creciente que suponía la animación del mercado era servida por una red de diligencias que unían la capital con Villajoyosa, Benidorm y Altea, en la ruta litoral septentrional; con Novelda y Elche en la ruta de Castilla; con Jijona, San Juan y San Vicente en su propio alfoz, y con la Vega Baja y la industriosa área alcoyana (13).

Todo ello supone una creciente especulación inmobiliaria, al calor de la expansión de la ciudad por las tierras adyacentes al antiguo núcleo murado y de las reformas interiores del casco, dando salida a los capitales acumulados en los negocios agromercantiles por la burguesía de la zona (14).

Mejoras de la accesibilidad para el área industrial alcoyana

Los valles alcoyanos se benefician también de la mejora generalizada de las 
comunicaciones. En este período se da por finalizada la carretera que une directamente la ciudad del Serpis con el puerto alicantino por la Carrasqueta y la Canal de Jijona. La solución de este enlace se había dilatado excesivamente en el tiempo, lastrando la circulación general de mercancías, servicios y personas entre ambas plazas. El periódico El Serpis, en 1878 denunciaba los perjuicios que para el puerto alicantino suponía esta situación (15), al producirse una fuga de tráficos hacia Gandía y Valencia.

Evidentemente, la secular mala accesibilidad de la hoya alcoyana ha jugado un papel secundario, pero real, en la génesis y evolución de ciertos atrasos técnicos que algunos de los sectores industriales de la zona padecieron durante gran parte de la centuria. No solamente existía un déficit a la hora de surtirse de fuentes de energía o dificultades en el proceso de acumulación de capital que permitiese una renovación tecnológica, sino también una distorsión de los circuitos de transporte que provoca una falta de agilidad notable en los canales comercializadores, lo que se traduce en una elevación constante de los costos de transferencia.

La etapa de la Restauración supone una mejora notable de la accesibilidad de la zona, no solamente por la terminación de la carretera a Alicante, sino también por la entrada en servicio de la de Cocentaina a Denia, por Pego, y por la mejora de los enlaces con el Alto Vinalopó, la Meseta y el altiplano de Yecla y Jumilla, completada con la inauguración, en la década de los noventa, del ferrocarril a Gandía.

Todo este contexto de mejoras en el transporte coincide cronológicamente con el auge de la industria alcoyana, hecho que se hace especialmente patente desde finales de los setenta hasta la década de los noventa. Así, en 1880 se instala la maquinaria Robert de fabricación de papel continuo en el taller Serra y en los primeros años noventa el textil sufre ajustes importantes en el proceso productivo, al aumentar la mecanización, con el consiguiente ascenso de la productividad y de los beneficios por un mayor volumen de producción y una reducción de la mano de obra.

De esta forma, en 1895, Vargas indicaba que la industria textil alcoyana era competitiva con la barcelonesa (16), aparte de que crecía también la papelera y la fabricación de cerillas y jabones y la fundición de hierro y construcción de maquinaria agrícola y calderería.

\section{Ferrocarril, carreteras y caminos y variaciones de la producción}

También la Hoya de Castalla presenta un sincronismo entre la mejora de su accesibilidad y el auge en la fabricación de las muñecas.

Al mismo tiempo, en los últimos años de la centuria, los municipios próximos al doble eje del ferrocarril y la carretera a Madrid en el Valle del Vinalopó ven aumentar su población y su capacidad de producción con un lento pero paulatino paso de la agricultura a la industria. 
Producciones de base agraria, como la fabricación de vinos y licores, toman un volumen considerable en aquellos núcleos que se conforman como encrucijadas de caminos en el corredor principal y, por ende, con un cierto desarrollo de la comercialización cual es el caso de Villena, Sax, Monóvar, Novelda y Aspe. En Elda, la fabricación del calzado empezaba a tener una cierta importancia (17), extendiéndose en mancha de aceite a los municipios próximos y bien comunicados de Petrer, Novelda y Aspe.

De esta forma nace y se desarrolla una industria dual en la comarca, hecho que ha llegado hasta nuestros días. Mientras que los núcleos situados en los valles occidentales marginales afluentes al corredor central quedarán atrapados en una agricultura predominantemente de secano y dominados por una fuerte atonía demográfica, los instalados en la zona directriz del esquema viario se revitalizan y se industrializan.

En el Bajo Vinalopó, Elche, favorecido por su situación dentro de la malla de transportes, dominando los flujos entre Murcia, Alicante y el resto del valle, desarrolla su industria alpargatera, de sacos y telares (18), al tiempo que Crevillente alcanza un alto grado de exportación de su producción de esteras.

\section{Principales logros viarios en el período}

La política de construcciones viarias en la provincia de Alicante durante la Regencia hay que enmarcarla en el contexto de la Ley de 11 de julio de 1877 que establece el tercer plan de carreteras del Ochocientos (19), en las constantes carencias de las Haciendas Públicas lo que supone abundantes atrasos y aplazamientos en la finalización de las obras, y en el electoralismo con el que se maneja el tema, con promesas y proyectos nunca llevados a cabo. Una Memoria de la Diputación de 1876 critica el hecho de que los caminos se diseñen e inicien sin tener presupuesto suficiente, con lo que las obras quedan frecuentemente paralizadas por impago a los contratistas (20).

A pesar de las dificultades, las construcciones viarias tuvieron un desarrollo notable, consiguiendo mejoras en todas las comarcas del territorio provincial alicantino (21).

El principal logro estriba en la mejora notable de la accesibilidad del área alcoyana. A fines de 1883 solamente quedaba por realizar un pequeño tramo de 133 metros en la arteria que une Alicante con Játiva por la ciudad del Serpis (22), aunque las lluvias producían fuertes daños en su estructura que obliga a realizar reparaciones en 1877, 1882 y 1888 (23). La carretera de Cocentaina a Denia por Pego estaba en construcción, siendo terminada a finales de la centuria, mientras que la del Barranco de la Batalla a Villajoyosa seguía en obras (24). El enlace de Alcoy con Callosa d'En Sarriá se estaba construyendo y la vía de Fuente la Higuera a Yecla por Caudete era proyectada en el tramo de siete kilómetros que faltaba para darle total continuidad (25). 
En La Marina, Pego se iba consolidando como un importante nudo de comunicaciones a medida que crecía la red viaria de la comarca. La carretera que la une con Oliva se ha concluido a fines de 1881 y se ha ensanchado (26), al tiempo que la arteria que la acerca al mar, enlazando con la nueva carretera de La Marina, se separa y reconstruye en los años setenta (27).

En 1882 se inician las obras de la carretera de Murla a Benissa por Jalón (28), al tiempo que se trabaja en la de Benidorm a Pego por Callosa (29).

La década de los ochenta va a ser decisiva para acabar el principal corredor de La Marina, la carretera de Alicante a Valencia por la costa, finalizándose el viaducto del Mascarat, faltando por construir algo más de diecisiete kilómetros en la zona valenciana entre Oliva y el río Molinell.

Al tiempo que en el área de la sierra Aitana se trabajaba entre 1886 y 1892 en distintos tramos de la vía que uniría Alcolecha con Penáguila (30), en el Campo de Alicante, muy carente de carreteras que estructuraran el territorio, continuaban las obras iniciadas en el Sexenio Revolucionario de reconstrucción y prolongación del enlace de Villafranqueza con la arteria a Játiva (31).

También en el Campo de Alicante se afronta la unión de los baños de Bussot con la carretera de Silla (32) y el enlace de San Vicente con la Hoya de Castalla, al tiempo que empieza a cristalizar el tema pendiente de la arteria que drenaría la costa meridional, desde Alicante a Torrevieja (33).

Durante el período, en el Valle del Vinalopó se desplegaba una importante actividad constructora. La carretera que en principio uniría la estación de Monóvar con Pinoso debía ser prolongada hacia Fortuna y sus baños y la estación de Archena. Dicho proyecto era tan vital para enlazar las zonas vitivinícolas a licantinas y murcianas, con el fin de introducirlas definitivamente en los circuitos comerciales capitalistas, que la Dirección General de Obras Públicas lo declaró preferente entre los de su clase. La Diputación lucentina ya había realizado en 1876 el tramo entre la estación de Monóvar y el pueblo y el que discurría entre el Pinoso y el límite de la provincia (34), estando en obras el intermedio entre ambas ciudades (35). Sin embargo la Diputación murciana no agilizaba el asunto, lo que dio motivo a una airada queja de la Corporación vecina, indicándose que esta actitud producía innumerables problemas a los vecinos del Pinoso, expresados de la siguiente forma:

«... ya que no se puede ir a los baños de Fortuna y Archena por las gentes de aquellos lugares ni sacar el grano y el vino» (36).

Para completar la vía citada, en 1866 se elaboraba el proyecto de carretera entre Monóvar y Novelda (37). Por las mismas fechas se trabaja en la de Aspe a Santa Pola, a través de Elche (38).

En la parte alta del Valle del Vinalopó existe un proyecto aprobado para realizar el camino de Biar a la Cañada y se planea el construir el tramo de carretera que ha de tocar el territorio provincial de la importante arterio que de las Casas 
del Campillo a Valencia, pasando por Onteniente, habría de ir a Villena. En 1877, al ayuntamiento de Bocairente solicita a la Diputación alicantina que active el estudio del trazado para poder conectar eficazmente con Alcudia de Crespins (39). Los años 1884 y 1887 fueron decisivos para la consecución de tal fin (40).

A fines de 1888 estaban prácticamente finalizadas las obras de un importante enlace del Valle del Vinalopó con el altiplano yeclano.

Por su parte, la Vega Baja del Segura veía también mejorar sus comunicaciones endógenas y exógenas. La carretera que uniría el Valle del Vinalopó, desde Novelda con Torrevieja, que no era más que la ampliación de la antiguamente proyectada entre Crevillente y Dolores, estaba parcialmente terminada a mediados de 1888 en el tramo Rojales-Torrevieja. Al tiempo, continuaban las obras en la de Elche a Dolores (41) y en la de Orihuela a la carretera de Torrevieja a Balsicas, que ya está terminada, por Bigastro y San Miguel de Salinas.

Los logros en la construcción de las carreteras durante el período fueron acompañados de una notable preocupación por la conservación de la red existente (42), destacando en este sentido las obras realizadas en las vías de Pego a Oliva (43), Gata a Jávea (44), Alcoy a Bañeres (45), Alicante a Agost (46) y Elche a Dolores (47). 
MAPA 1

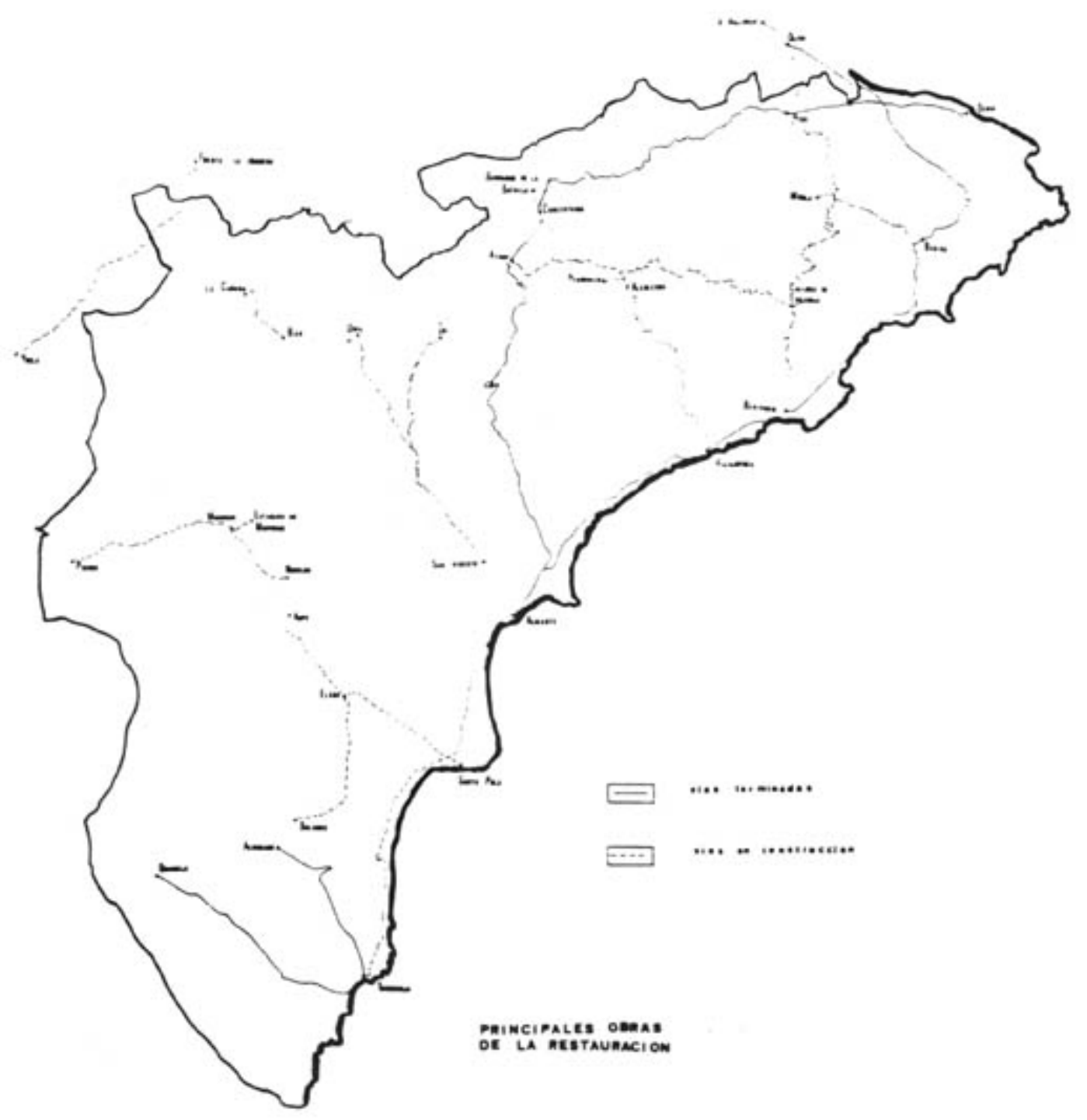




\section{MAPA 2}

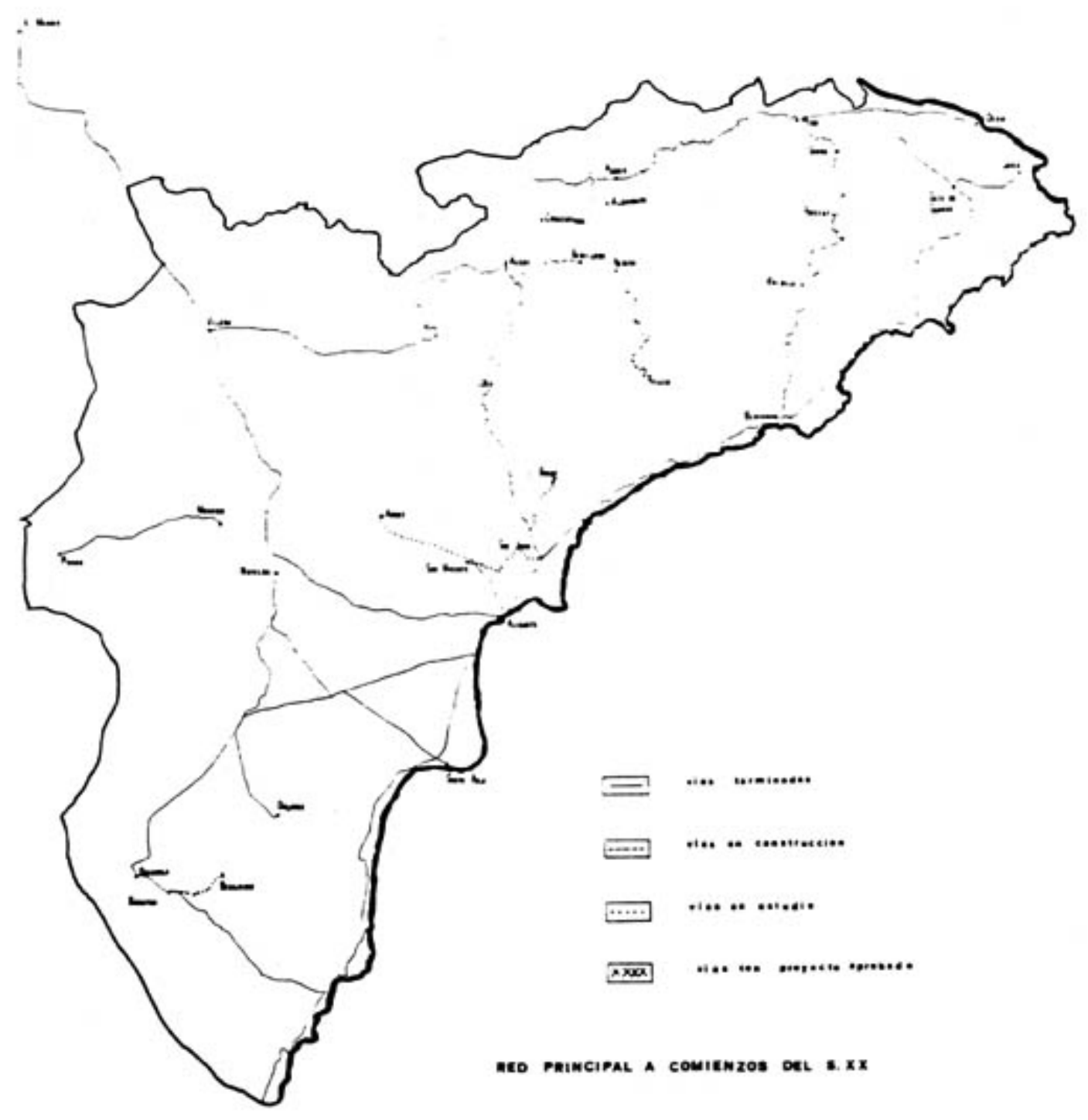




\section{NOTAS}

1. Por lo que hace referencia a la industrialización vid. FERRÁNDIZ PONZO, J., Estado de la industria de la provincia de Alicante e indicación de las nuevas industrias que podrían implantarse en ella, o cuando menos, en algunas localidades. Alicante, J. Bernabeu, 1901.

2. En la prensa de la época es frecuente el reclamar una agilización en las construcciones viarias con este fin. Vid La voz de Jijona, de 26 de enero de 1879.

3. ECHEVARRÍA, A., Memoria sobre el estado de la agricultura en la provincia de Alicante. Archivo Diputación Provincial (ADP). Sección Fomento. Leg. 120. Está fechada en 1875.

4. OROZCO SÁNCHEZ, O. P., Manual geográfico-estadístico de la provincia de Alicante. Alicante, Imprenta de Antonio Reus, 1878.

5. Ibid. p. 267.

6. OBRAS PÚBLICAS, Memoria sobre las Obras Públicas en el año de 1882, comprendiendo lo relativo a carreteras, presentada al Excmo. Sr. Ministro de Fomento por el Excmo. Sr. Don Gabriel Enríquez, director general de Obras Públicas. Madrid, Tipografía Hispano Americana, 1884.

7. OBRAS PÚBLICAS, Memoria sobre el estado de las carreteras en el año 1888 presentada al Excmo. Sr. Ministro de Fomento por el Ilmo. Sr. Don Manuel Pardo y Sánchez Salvador, director general interino de Obras Públicas. Madrid, Tipografía de Ricardo Álvarez, 1889.

8. MINISTERIO DE FOMENTO, Presupuesto de 1897-1898. Balance General de créditos y gastos y monografías, consideradas bajo su parte económica, de todas las obras y servicios durante el expresado ejercicio en los ramos de Instrucción Pública, Agricultura, Industria, Comercio, Obras Públicas e Instituto Geográfico y Estadístico. Madrid, Romero Impresor, 1899.

9. OBRAS PÚBLICAS, Estadística de las Obras Públicas en 1901. Madrid, Establecimiento Tipográfico de Hijos de J. A. García, 1901.

10. A. D. P., Sec. II, Leg. 38.

11. VARGAS, J. de, Viaje por España: Alicante y Murcia. Madrid, El Liberal, 1895, p. 149.

12. GOZÁLVEZ, V., «La población», apud LÓPEZ GÓMEZ, A. et al., Geografía de la provincia de Alicante. Alicante, Diputación Provincial, 1978, p. 189.

13. RAMOS, V., Historia de la provincia de Alicante y de su capital. Alicante, Diputación Provincial, 1971, T. II, p. 20. 
14. Para verla expansión de la ciudad, vid. RAMOS, A., Evolución urbana de Alicante. Alicante, Instituto de Estudios Juan Gil Albert, Diputación Provincial, 1984, pp. 217-224.

15. El Serpis, de 9 de mayo de 1878.

16. VARGAS, J. de, Opus cit., pp. 172-177.

17. Vid. AlMiÑANA, N., Guía de las provincias de Alicante y Murcia y Crónica-Indicador de Alcoy para el año 1887. Alicante, Costa y Mira, 1886.

18. IBARRA Y RUIZ, P., Historia de Elche. Alicante, Tip. V. Botella, 1895. pp. 293-295 y 300.

19. Previo al plan de carreteras, se promulgaron la Ley General de Obras Públicas, de 13 de abril de 1877 y la Ley General de Carreteras, de 4 de mayo del mismo año.

20. A. D. P., Memoria presentada por la Comisión Permanente a la Excelentísima Diputación Provincial. Manuscrita, fechada a 1 de abril de 1876. Esta situación no era más que un fiel reflejo a escala provincial de lo que ocurría a nivel nacional. Vid. «Un alto ejemplo de inmoralidad: las carreteras parlamentarias», Revista de Obras Públicas, n 1, 255, Madrid, 2 de octubre de 1899, pp. 389-391.

21. Una importante fuente documental en A. D. P.: Libro de Registro de Certificaciones de Obras, especificando el capataz al que se adjudican, comprendiendo desde 1883 a 1920, Sec. II, leg. 442.

22. OBRAS PÚBLICAS, Memoria sobre el estado de las carreteras en el año de 1883 presentada al Excmo. Sr. Ministro de Fomento por el Excmo. Sr. Don Mariano Catalina y Cobo, director general de Obras Públicas. Madrid, Tipografía de los Huérfanos, 1886.

23. A. D. P., Sec. II, leg. 259.

24. Las obras de esta última vía fueron lentas en el período 1875-1877, intensificándose en los años siguientes, subastándose el trozo tercero en 1881, según consta en A. D. P., sec. II. leg. 163, 16, $22,179,277$ y 298 por lo que hace referencia a las realizaciones; leg. 70 y 260 por lo que concierne a los proyectos y 142, 212, 266, 271, 328 y 337 para las reparaciones. Vid., también, Memoria de la Diputación, manuscrita, fechada el 31 de marzo de 1881.

25. OBRAS PÚBLICAS, Memoria sobre el estado de las carreteras en el año 1885, presentada al Excmo. Sr. Ministro de Fomento por el Excmo. Sr. Don José Gallego Díaz, director general de Obras Públicas. Madrid, Tipo-Litografía de L. Peant e Hijos, 1887.

26. A. D. P., Secc. II, leg. 70.

27. A D. P., Secc. II, leg. 9, con los expedientes de expropiación y leg. 90 y 379, con los de obras.

28. OBRAS PÚBLICAS, Opus cit. not. 6.

29. Ibid. y MINISTERIO DE FOMENTO, Opus cit., not. 8.

30. A. D. P., Secc. II, leg. 216.

31. A. D. P., Secc. II, leg. 99, 165, 234 y 254.

32. La mitad del trazado se abre al tráfico el 24 de julio de 1899.

33. Por Ley de 17 de julio de 1885, se comienza a estudiar el tramo Altet-Torrevieja y el 4 de enero de 1899 se subastan las obras del trozo Alicante-El Altet.

34. A D. P., Secc. II, leg. 231.

35. A. D. P., Secc. II, leg. 65 y 162. Durante los años setenta y ochenta se realizaron múltiples 
obras de conservación de esta carretera, tal como consta en leg. 182, 209, 259, 274 y 236.

36. A. D. P., Memoria presentada por la Comisión Permanente a la Excelentísima Diputación Provincial, manuscrita, fechada en Alicante, a 31 de octubre de 1876.

37. A. D. P., Secc. II, leg. 245.

38. A fines de 1888 estaba terminado totalmente el trozo primero.

39. A. D. P., Memoria de la Diputación, manuscrita, fechada en noviembre de 1877.

40. Por Real Decreto de 16 de agosto de 1884 se autoriza al Ministerio de Fomento la subasta del trozo primero de la provincia de Alicante, con una longitud de 7,8 km., adjudicándose la obra el 23 de octubre. Mientras se trabajaba en este tramo, el 19 de diciembre de 1877 se adjudican los tramos segundo y tercero, iniciándose las actuaciones en mayo de 1888.

41. A. D. P., Secc. II, leg. 228.

42. Un gran número de expedientes sobre conservación de carreteras y caminos en A. D. P., Secc. II, leg. 166.

43. A. D. P., Secc. II, leg. 99, 259, 211, 266 у 272.

44. A. D. P., Secc. II, leg. 98 y 259.

45. A. D. P., Secc. II, leg. 208, 266 y 273.

46. A. D. P., Secc. II, leg. 487.

47. A. D. P., Secc. II, leg. 71, 207, 259, 276 y 390. 